\title{
Kinematic modeling and verification for a SCARA robot
}

\author{
Chenhuan Feng ${ }^{1,}$, Guanbin Gao ${ }^{1, b}$, Yongli Cao ${ }^{2, c}$ \\ ${ }^{1}$ Faculty of Mechanical and Electrical Engineering, Kunming University of Science and Technology, \\ Kunming 650500, China \\ ${ }^{2}$ Kunming Yunnei Power Co., Ltd, Kunming Economic Development Zone No. 66, King Road, \\ Kunming 650027, China
}

bfengchenhuan-sjz@163.com, agggao@163.com, ${ }^{\mathrm{c} y l}$ _cao@sina.com

Keywords: Kinematic model, SCARA robot, DH method, Simulation

\begin{abstract}
The kinematic model of robots is to describe the nonlinear relationship between the position and orientation of the end-effector and the displacement of each joint, which is an important content of robot calibration. The coordinate systems of a SCARA robot are established according to DH method, through which the structural parameters are determined. Then, the kinematic equations between the working space and the joint space are deduced by homogeneous transformation principle. The graphic and calculating model of the SCARA robot are established in Matlab. Through the comparison between graphic and calculating model, the kinematic model is verified.
\end{abstract}

\section{Introduction}

SCARA robot is a kind of special cylindrical coordinate type of industrial robots, which relies on two rotary joints for rapid positioning within the horizontal plane as well as a linear joint and a rotary joint for rotary and stretchy movement within the $\mathrm{Z}$ direction. The structure of the SCARA robot studied in this paper is shown in Fig. 1. It's movement is smooth and reliable in the horizontal direction, and it has a greater stiffness in the vertical direction [1]. In order to improve the accuracy of the robot, kinematic parameters calibration must be done first. Furthermore, kinematic modeling is the theoretical foundation of calibration. Through a function relationship between the end-effector position, orientation matrix and the joint variables, we can determine the parameters of the adjacent coordinate system to obtain a homogeneous transformation relationship. Finally, we can get the kinematic model of the robot using multiplication[2]. The purpose of the establishment of the kinematic model is to find the original error of each parameter transfer coefficient for the results influence, as well as synthetic relationship between the various errors.

SCARA robot movement usually needs to be high-speed and high-precision. Therefore, the analysis of the robot kinematics must be careful, accurate and efficient[3]. Due to the DH model has clear physical meaning and easy programming, this paper established the kinematic model with DH model method, and Matlab simulation was used to verify the model.

\section{Coordinate systems of the robot based on DH model}

Denavit-Hertenberg method, referred to as DH method, is now the most commonly used method of robot kinematic model, which uses a homogeneous transformation matrix to describe the relative position between two adjacent links[4]. The reference coordinate system on the base should be established first in this paper. Then, we established four joint coordinate systems of No.1 to 4 . The reference coordinate system is stationary in the working process of the SCARA robot, and others coordinate systems move with the corresponding joints. In addition, the kinematic equation is the product of the coordinate transformation[5].

Method of establishing the coordinate system of each joint are as follows:

(1) Determining the $z_{i}$ axis, the $z_{i}$ axis is along the axis direction of the joint $z_{i+1}$;

(2) Determining the origin $o_{i}$, the $o_{i}$ is on common perpendicular of $z_{i}$ and $z_{i-1}$ axis, intersection of the common perpendicular and axis $z_{i}$ is the origin $o_{i}$; 
(3) Determining the $x_{i}$ axis, the $x_{i}$ axis is along the direction of the common perpendicular of $z_{i}$ and $z_{i-1}$ axis and leaving the $z_{i-1}$ axis;

(4) Determining the $y_{i}$ axis with the right hand rule.

The coordinate systems of the SCARA robot established with DH method are shown as Fig. 2.
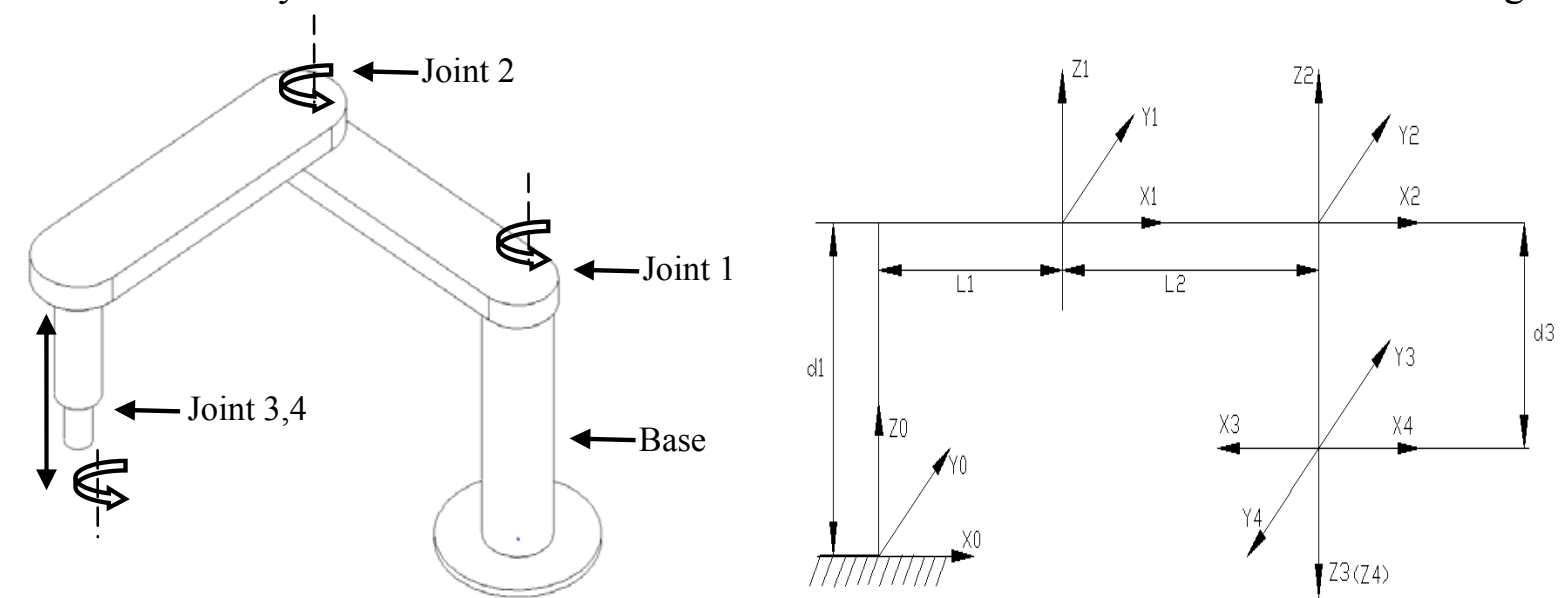

Fig. 1 The structure of the SCARA robot

Fig. 2 The coordinate systems of the SCARA robot

According to the principle of $\mathrm{DH}$ method, there are four groups structural parameters of the SCARA robot[6]. They are, respectively, linkage length $d_{i}$, joint length $a_{i}$, torsion angle $\alpha_{i}$ and joint angle $\theta_{i}$. These parameters are defined as follows:

(1) $d_{i}$ is the translation between the axis of $x_{i-1}$ and $x_{i}$, along $z_{i}$ direction is positive;

(2) $a_{i-1}$ is the translation between the axis of $z_{i-1}$ and $z_{i}$, along $x_{i-1}$ direction is positive;

(3) $\alpha_{i-1}$ is the rotation between the axis of $z_{i-1}$ and $z_{i}$, about $x_{i-1}$ counter-clockwise;

(4) $\theta_{i}$ is the rotation between the axis of $x_{i}$ and $x_{i-1}$, about $z_{i}$ counter-clockwise;

The DH parameters of the SCARA robot are shown in Table 1, where $\theta_{i}$ and $\mathrm{d} 3$ is variable; L1 $=$ $250 \mathrm{~mm}$; $\mathrm{L} 2=350 \mathrm{~mm} ; \mathrm{d} 1=300 \mathrm{~mm}$.

Table 1. The DH parameters of SCARA robot

\begin{tabular}{ccccc}
\hline Joints & $d_{i}(\mathrm{~mm})$ & $a_{i-1}(\mathrm{~mm})$ & $\alpha_{i-1}\left({ }^{\circ}\right)$ & $\theta_{i}\left({ }^{\circ}\right)$ \\
\hline 1 & $\mathrm{~d} 1$ & $\mathrm{~L} 1$ & 0 & $\theta_{1}$ \\
2 & 0 & $\mathrm{~L} 2$ & 0 & $\theta_{2}$ \\
3 & $-\mathrm{d} 3$ & 0 & 180 & 0 \\
4 & 0 & 0 & 0 & $\theta_{4}$ \\
\hline
\end{tabular}

\section{Kinematic equations of the robot}

According to the coordinate systems and homogeneous transformation method, the homogeneous transformation matrix of the SCARA robot can be determined from (1):

$$
\begin{aligned}
T_{i-1, i} & =\operatorname{Rot}\left(z_{i-1}, \theta_{i}\right) \operatorname{Trans}\left(0,0, d_{i}\right) \operatorname{Trans}\left(a_{i}, 0,0\right) \operatorname{Rot}\left(x_{i}, \alpha_{i}\right) \\
& =\left[\begin{array}{cccc}
\cos \theta_{i} & -\sin \theta_{i} \cos \alpha_{i} & \sin \theta_{i} \sin \alpha_{i} & a_{i} \cos \theta_{i} \\
\sin \theta_{i} & \cos \theta_{i} \cos \alpha_{i} & -\cos \theta_{i} \sin \alpha_{i} & a_{i} \sin \theta_{i} \\
0 & \sin \alpha_{i} & \cos \alpha_{i} & d_{i} \\
0 & 0 & 0 & 1
\end{array}\right] .
\end{aligned}
$$

We obtained pose transformation matrix between two adjacent coordinates through the DH parameters of table 1, they are shown in (2) and (3): 


$$
\begin{aligned}
T_{0,1} & =\left[\begin{array}{cccc}
\cos \theta_{1} & -\sin \theta_{1} & 0 & L 1 \cos \theta_{1} \\
\sin \theta_{1} & \cos \theta_{1} & 0 & L 1 \sin \theta_{1} \\
0 & 0 & 1 & d_{1} \\
0 & 0 & 0 & 1
\end{array}\right], T_{1,2}=\left[\begin{array}{cccc}
\cos \theta_{2} & -\sin \theta_{2} & 0 & L 2 \cos \theta_{2} \\
\sin \theta_{2} & \cos \theta_{2} & 0 & L 2 \sin \theta_{2} \\
0 & 0 & 1 & 0 \\
0 & 0 & 0 & 1
\end{array}\right] . \\
T_{2,3} & =\left[\begin{array}{cccc}
1 & 0 & 0 & 0 \\
0 & -1 & 0 & 0 \\
0 & 0 & -1 & -d_{3} \\
0 & 0 & 0 & 1
\end{array}\right], T_{3,4}=\left[\begin{array}{cccc}
\cos \theta_{4} & -\sin \theta_{4} & 0 & 0 \\
\sin \theta_{4} & \cos \theta_{4} & 0 & 0 \\
0 & 0 & 1 & 0 \\
0 & 0 & 0 & 1
\end{array}\right] .
\end{aligned}
$$

The pose equation of End-effector of the SCARA robot was obtained by multiplying transformation matrix of each joint, it can be shown in (4):

$$
\begin{aligned}
T_{0,4}=T_{0,1} T_{1,2} T_{2,3} T_{3,4} & =\left[\begin{array}{cccc}
\cos \left(\theta_{1}+\theta_{2}-\theta_{4}\right) & -\sin \left(\theta_{1}+\theta_{2}-\theta_{4}\right) & 0 & L 1 \cos \theta_{1}+L 2 \cos \left(\theta_{1}+\theta_{2}\right) \\
\sin \left(\theta_{1}+\theta_{2}-\theta_{4}\right) & \cos \left(\theta_{1}+\theta_{2}-\theta_{4}\right) & 0 & L 1 \sin \theta_{1}+L 2 \sin \left(\theta_{1}+\theta_{2}\right) \\
0 & 0 & -1 & d 1-d 3 \\
0 & 0 & 0 & 1
\end{array}\right] \\
& =\left[\begin{array}{cccc}
n_{x} & o_{x} & a_{x} & p_{x} \\
n_{y} & o_{y} & a_{y} & p_{y} \\
n_{z} & o_{z} & a_{z} & p_{z} \\
0 & 0 & 0 & 1
\end{array}\right] .
\end{aligned}
$$

If given joint parameters, we can calculate the robot end-effector position $\left(\mathrm{p}_{\mathrm{x}}, \mathrm{p}_{\mathrm{y}}, \mathrm{p}_{\mathrm{z}}\right)$ on the reference coordinate system $\left\{\mathrm{X}_{0} \mathrm{Y}_{0} \mathrm{Z}_{0}\right\}$.

\section{Robot kinematic simulation based on Matlab}

A simulation is important for robot programmers because it can allow them to evaluate and predict the behavior of a robot, in addition it also can verify and optimize the path planning of process [7]. From the above content, the kinematic model of four free SCARA robot was obtained through the establishment of five coordinate systems and four coordinate transformations, the process of solving the coordinate system and structure parameters are very complex. This paper adopted the method of numerical calculation and graphic simulation which verifies the robot kinematic model, verification process is shown in Fig. 3.

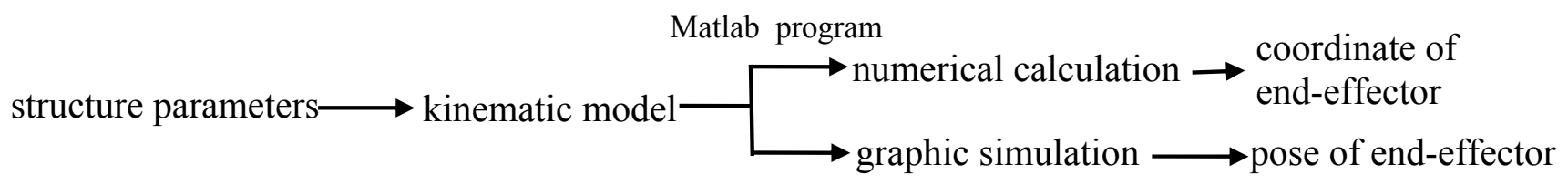

Fig. 3 The validation flowchart of the kinematic model

According to the homogeneous transformation matrix (2), (3) and (4), we wrote a numerical calculation program in Matlab, its result is verified by the graphic simulation [8]. Depending on the structural parameters, coordinate values of the end-effector are calculated in the reference coordinate system.

Robotics toolbox is developed by Corke in the Matlab platform for robot kinematics and dynamics, which has the characteristics of intuitive and simple programming. In this paper, the Robotics toolbox was used for robot graphic simulation, the simulation program was written by $\mathrm{m}$ file. Eventually,we can achieve kinematic data output of the robot.

The key part of $\mathrm{m}$ file program is shown below:

$\% \quad$ alpha $\left(\alpha_{i}\right) \quad \mathrm{a}\left(a_{i}\right)$ theta $\left(\theta_{i}\right)$ d $\left(d_{i}\right)$ sigma offset

$\mathrm{L} 1=\operatorname{link}\left(\left[\begin{array}{llllll}0 & 250 & 0 & 300 & 0 & 0\end{array}\right]\right) ;$ 


$\begin{array}{lccccc}\mathrm{L} 2=\operatorname{link}([0 & 350 & 0 & 0 & 0 & 0]) ; \\ \mathrm{L} 3=\operatorname{link}([\mathrm{pi} & 0 & 0 & 0 & 1 & 0]) ; \\ \mathrm{L} 4=\operatorname{link}([0 & 0 & 0 & 0 & 0 & 0]) ;\end{array}$

AACMM=robot $(\{$ L1 L2 L3 L4 $\})$;

Where, sigma is joint type, rotary joint is 0 , linear joint is 1 ; offset is joint variable offset. The initial position (at $\mathrm{t}=0$ ) is the home position of SCARA robot where $\theta_{1}=0^{\circ}, \theta_{2}=0^{\circ}, \theta_{4}=0^{\circ}$ and $\mathrm{d} 3=0 \mathrm{~mm}$.

In order to verify the five different poses in this paper, the coordinates of the end-effector were calculated with the numerical simulation program as shown in Table 2. We gained simulation figure and coordinate values of the five different poses by the graphics simulation program. Compared the results of two methods, we found they were completely identical.

Table 2. The result of numerical simulation

\begin{tabular}{ccl}
\hline Group & Pose $\left(\theta_{1}, \theta_{2}, \mathrm{~d} 3, \theta_{4}\right)$ & coordinate \\
\hline 1 & $\left(-180^{\circ}, 0,0,0\right)$ & $(-600,0,300)$ \\
2 & $\left(-180^{\circ},-90^{\circ}, 0,0\right)$ & $(-250,350,300)$ \\
3 & $\left(-180^{\circ},-90^{\circ}, 100,0\right)$ & $(-250,350,200)$ \\
4 & $\left(-180^{\circ},-90^{\circ}, 100,90^{\circ}\right)$ & $(-250,350,200)$ \\
5 & $\left(90^{\circ},-90^{\circ}, 100,90^{\circ}\right)$ & $(350,250,200)$ \\
\hline
\end{tabular}

\section{Conclusion}

The modeling methods for industrial robots were studied in this paper. The first step was to establish the coordinate systems of each joint of the robot with the DH method. Then, according to the mathematical derivation of kinematic system and homogeneous transformation method, the kinematic model was analyzed. Finally, the kinematic model of the robot was verified through the Matlab software program. The robot kinematic model is composed of its kinematic equation, which can describe the pose relation between each joint and end-effector of the robot in the working space.

The kinematic modeling method used in this paper also can be applied to other similar robots.

\section{Acknowledgements:}

The authors would like to thank Yunnan Provincial Natural Science Foundation of China (Grant No.2011FB028). The email of the corresponding author is gbgao@163.com.

\section{References}

[1] L. Shi, Y.C Jiang, Z.Q. Jing: ICMPMT 2011. Vol. 338 (2011), p. 766.

[2] Y.J. Zhao, S.N. Yang: IEEE TRANSACTIONS ON ROBOTICS. Vol. 26 (2010), No. 3, p. 414.

[3] M. Al, I. Yousif: IRAM 2012. Vol. 330 (2012), p. 94.

[4] J. Denavit, and R. Hartenberg: ASME Journal of Applied Mechnics Vol. 22(1955), No. 6, p. 215.

[5] G.B. Gao, J. Lu, J.J. Zhou: Advanced Materials Research Vol. 11(2012), No.590, p 471.

[6] I.M. Chen, G.L. Yang: Mechanism and Machine Theory Vol. 36(2001), No.11-12, p.1215.

[7] G. Mahmoud, O. Mohammed: Int J Adv Robotic Sy. Vol. 9(2012), No. 245, p. 332.

[8] G.D. Fu, X.Z. Zheng, S. Huang: Journal of Mechanical Transmission. Vol. 39(2015), No. 9, p. 151. 